\title{
Super-biderivations of Cartan type Lie superalgebras
}

\author{
Jixia Yuan, ${ }^{1,2, *}$ Liangyun $\mathrm{Chen}^{1, \dagger}$ and YAN CaO ${ }^{3, \ddagger}$ \\ 1. School of Mathematical and Statistics, Northeast Normal University \\ Changchun 130024, China
}

2. School of Mathematical Sciences, Heilongjiang University Harbin 150080, China

3. Department of Mathematics, Harbin University of Science and Technology Harbin 150080, China

\begin{abstract}
In this paper, we characterize the super-biderivations of Cartan type Lie superalgebras over the complex field $\mathbb{C}$. Furthermore, we prove that all super-biderivations of Cartan type simple Lie superalgebras are inner superbiderivations.
\end{abstract}

Mathematics Subject Classification. 17B40, 17B65, 17B66.

Keywords. Lie superalgebras, Cartan type Lie superalgebras, super-derivations, super-biderivations.

\section{Introduction}

As is well known, all the finite dimensional simple Lie superalgebras over $\mathbb{C}$ consist of classical Lie superalgebras and Cartan type Lie superalgebras [9]. However, four families of $\mathbb{Z}$-graded Lie superalgebras of Cartan-type are, the Witt type, the special type and the Hamiltonian type. These Lie superalgebras are subalgebras of the full superderivation algebras of the exterior superalgebras. The structural theory of these superalgebras have been playing a key role in the theory of Lie superalgebras. Derivations and generalized derivations are very important subjects in the research of both algebras and their generalizations.

The concept of biderivations were introduced and studied in [1], and the authors showed that all biderivations on commutative prime rings are inner biderivations and determined

\footnotetext{
* Supported by the National Natural Science Foundation of China (11601135), Natural Science Foundation of Heilongjiang Province of China (QC2017002) and Project funded by China Postdoctoral Science Foundation

†Correspondence: chenly640@nenu.edu.cn. (L. Chen); Supported by the National Natural Science Foundation of China (11771069, NSF of Jilin province (No. 20170101048JC) and the project of jilin province department of education (No. JJKH20180005K))

$\ddagger$ Supported by the National Natural Science Foundation of China (11801121), Natural Science Foundation of Heilongjiang Province of China (QC2018006)
} 
the biderivations of semiprime rings. In recent years, biderivations have aroused the interest of a great many authors, see [1, 4, [6]. The notion of biderivations of Lie algebras was introduced in [14]. The biderivations of Lie algebras have been sufficiently studied. In [2, 3, 15], the authors proved that all anti-symmetric biderivations on the SchrödingerVirasoro algebra, the simple generalized Witt algebra and the infinite-dimensional Galilean conformal algebra are inner, respectively. In [7], the authors determined all the skewsymmetric biderivations of $\mathrm{W}(\mathrm{a} ; \mathrm{b})$ and found that there exist non-inner biderivations. In [12, 13], the authors characterized the biderivations without the anti-symmetric condition of the finite dimensional complex simple Lie algebra and some W-algebras, meanwhile, presented some classes of non-inner biderivations. In 2015, Xu and Wang generalized the notion of biderivations of Lie algebra to the super case in [16], and the authors mainly discussed some properties of super-biderivations on Heisenberg superalgebras. In [5], the authors proved that all super-skewsymmetric super-biderivations on the centerless superVirasoro algebras are inner super-biderivations. In [17], the authors proved that any superskewsymmetric super-biderivation of super-Virasoro algebras was inner. In [18], the authors proved that all super-biderivations of classical simple Lie superalgebras were inner superbiderivations and finded a class of a non-inner and non-skewsymmetric super-biderivations of general linear Lie superalgebra. In [10], the authors determined all the biderivations of $W(2,2)$ and found that there existed non-inner biderivations.

In this paper, we are interested in determining all super-biderivations of Cartan type Lie superalgebras over $\mathbb{C}$ without the skew-symmetric condition. The main result in this paper is a complete characterization of the super-biderivations of Cartan type Lie superalgebras. The paper is organized as follows. In Section 2, we recall some necessary concepts and notations. In Section 3, we establish several lemmas, which will be used to characterize the super-biderivations of classical simple Lie superalgebras. In Section 4, we determine all super-biderivations of Cartan type Lie superalgebras $W(n), S(n), \widetilde{S}(n)$ without the superskewsymmetric condition. In Section 5, we determine all super-biderivations of Cartan type Lie superalgebras $H(n)$ without the super-skewsymmetric condition.

\section{Preliminaries}

Throughout $\mathbb{C}$ is the field of complex numbers, $\mathbb{N}$ the set of nonnegative integers and $\mathbb{Z}_{2}=\{\overline{0}, \overline{1}\}$ the additive group of two elements. For a vector superspace $V=V_{\overline{0}} \oplus V_{\overline{1}}$, we write $|x|$ for the parity of $x \in V_{\alpha}$, where $\alpha \in \mathbb{Z}_{2}$. Once the symbol $|x|$ appears in this paper, it will imply that $x$ is a $\mathbb{Z}_{2}$-homogeneous element. We also adopt the following notation: For a proposition $P$, put $\delta_{P}=1$ if $P$ is true and $\delta_{P}=0$ otherwise.

\subsection{Lie algebras, derivation and biderivation}

Let us recall some definitions relative to Lie algebras [8]. A Lie algebra is a vector space $L$ with a bilinear mapping $[\cdot, \cdot]: L \times L \longrightarrow L$ satisfying the following axioms:

$$
\begin{aligned}
& {[x, y]=-[y, x]} \\
& {[x,[y, z]]=[[x, y], z]+[y,[x, z]]}
\end{aligned}
$$

for all $x, y, z \in L$.

We call a bilinear map $f: L \times L \longrightarrow L$ a biderivation of $L$ if it satisfies the following 
two equations:

$$
\begin{aligned}
& f([x, y], z)=[x, f(y, z)]+[f(x, z), y] \\
& f(x,[y, z])=[f(x, y), z]+[y, f(x, z)]
\end{aligned}
$$

for all $x, y, z \in L$. If the map $f_{\lambda}: L \times L \longrightarrow L$, defined by $f_{\lambda}(x, y)=\lambda[x, y]$ for all $x, y \in L$, where $\lambda \in \mathbb{C}$, then it is easy to check that $f_{\lambda}$ is a biderivation of $L$. We call this class of biderivations by inner biderivations.

\subsection{Lie superalgebras, super-derivation and super-biderivation}

Let us recall some definitions relative to Lie superalgebras [9]. A Lie superalgebra is a vector superspace $L=L_{\overline{0}} \oplus L_{\overline{1}}$ with an even bilinear mapping $[\cdot, \cdot]: L \times L \longrightarrow L$ satisfying the following axioms:

$$
\begin{aligned}
& {[x, y]=-(-1)^{|x||y|}[y, x]} \\
& {[x,[y, z]]=[[x, y], z]+(-1)^{|x||y|}[y,[x, z]]}
\end{aligned}
$$

for all $x, y, z \in L$.

We call a linear map $D: L \longrightarrow L$ a super-derivation of Lie superalgebra $L$ if it satisfies the following equation:

$$
D([x, y])=[D(x), y]+(-1)^{|D||x|}[x, D(y)]
$$

for all $x, y \in L$. Write $\operatorname{Der}_{\overline{0}} L$ (resp. $\operatorname{Der}_{\overline{1}} L$ ) for the set of all super-derivations of $\mathbb{Z}_{2^{-}}$ homogeneous $\overline{0}$ (resp. $\overline{1}$ ) of $L$. Denote

$$
\operatorname{Der} L=\operatorname{Der}_{\overline{0}} L \oplus \operatorname{Der}_{\overline{1}} L .
$$

We call a bilinear map $f: L \times L \longrightarrow L$ a super-biderivation of $L$ if it satisfies the following two equations:

$$
\begin{aligned}
& f([x, y], z)=(-1)^{|x||f|}[x, f(y, z)]+(-1)^{|y||z|}[f(x, z), y] \\
& f(x,[y, z])=[f(x, y), z]+(-1)^{(|f|+|x|)|y|}[y, f(x, z)]
\end{aligned}
$$

for all $x, y, z \in L$. Note that our definition of super-biderivation does not assume particular super-skewsymmetry which is different from [5]. If the map $f_{\lambda}: L \times L \longrightarrow L$ is defined by $f_{\lambda}(x, y)=\lambda[x, y]$ for all $x, y \in L$, where $\lambda \in \mathbb{C}$, then it is easy to check that $f_{\lambda}$ is a super-biderivation of $L$. This class of super-biderivations is said to be inner. Denote by $\operatorname{IBDer}(L)$ the set of all inner super-biderivations.

A super-biderivation $f$ of $\mathbb{Z}_{2}$-homogeneous $\gamma$ of $L$ is a super-biderivation such that $f\left(L_{\alpha}, L_{\beta}\right) \subseteq L_{\alpha+\beta+\gamma}$ for any $\alpha, \beta \in \mathbb{Z}_{2}$. Write $\operatorname{BDer}_{\overline{0}}(L)$ (resp. $\operatorname{BDer}_{\overline{1}}(L)$ ) for the set of all super-biderivations of $\mathbb{Z}_{2}$-homogeneous $\overline{0}$ (resp. $\overline{1}$ ) of $L$. Denote

$$
\operatorname{BDer}(L)=\operatorname{BDer}_{\overline{0}}(L) \oplus \operatorname{BDer}_{\overline{1}}(L) .
$$

\subsection{Cartan type Lie superalgebras}

Let $n \geq 4$ be an integer and $\Lambda(n)$ be the exterior algebra in $n$ indeterminates $x_{1}, x_{2}, \ldots, x_{n}$ with $\mathbb{Z}_{2}$-grading structure given by $\left|x_{i}\right|=\overline{1}$. One may define a $\mathbb{Z}$-grading on $\Lambda(n)$ by letting $\operatorname{deg} x_{i}=1$, where $1 \leq i \leq n$. Write $n=2 r$ or $n=2 r+1$, where $r \in \mathbb{N}$. Put $\left[\frac{n}{2}\right]=r$. Cartan 
type Lie superalgebras consist of four series of simple Lie superalgebras contained in the full superderivation algebras of $\Lambda(n)$ :

$$
\begin{aligned}
& W(n)=\left\{\sum_{i=1}^{n} f_{i} \partial_{i} \mid f_{i} \in \Lambda(n)\right\} \\
& S(n)=\left\{\sum_{i=1}^{n} f_{i} \partial_{i} \mid f_{i} \in \Lambda(n), \sum_{i=1}^{n} \partial_{i}\left(f_{i}\right)=0\right\}, \\
& \widetilde{S}(n)=\left\{\left(1-x_{1} x_{2} \cdots x_{n}\right) \sum_{i=1}^{n} f_{i} \partial_{i} \mid f_{i} \in \Lambda(n), \sum_{i=1}^{n} \partial_{i}\left(f_{i}\right)=0\right\}(n \text { is an even integer }), \\
& H(n)=\left\{\mathrm{D}_{\mathrm{H}}(f) \mid f \in \oplus_{i=0}^{n-1} \Lambda(n)_{i}\right\} \quad(n>4),
\end{aligned}
$$

where

$$
\begin{gathered}
\mathrm{D}_{\mathrm{H}}(f)=(-1)^{|f|} \sum_{i=1}^{n} \partial_{i}(f) \partial_{i^{\prime}}, \\
i^{\prime}= \begin{cases}i+r, & \text { if } 1 \leq i \leq\left[\frac{n}{2}\right], \\
i-r, & \text { if }\left[\frac{n}{2}\right]<i \leq 2\left[\frac{n}{2}\right], \\
i, & \text { otherwise. }\end{cases}
\end{gathered}
$$

A simple computation shows

$$
\left[\mathrm{D}_{\mathrm{H}}(f), \mathrm{D}_{\mathrm{H}}(g)\right]=\mathrm{D}_{\mathrm{H}}\left(\mathrm{D}_{\mathrm{H}}(f)(g)\right) \text { for } f, g \in \Lambda(n) \text {. }
$$

One may define a $\mathbb{Z}$-grading on $W(n)$ by letting $\operatorname{deg} x_{i}=1=-\operatorname{deg} \partial_{i}$, where $1 \leq i \leq n$. Thus $W(n)$ becomes a $\mathbb{Z}$-graded Lie superalgebra of 1 depth: $W(n)=\bigoplus_{j=-1}^{\xi_{W}} W(n)_{j}$, where $\xi_{W}=n-1$. Suppose $L=S(n)$ or $H(n)$. Then $L$ is a $\mathbb{Z}$-graded subalgebra of $W(n)$. The $\mathbb{Z}$-grading is defined as follows: $L=\bigoplus_{j=-1}^{\xi_{L}} L_{j}$, where $L_{j}=L \cap W(n)_{j}$ and

$$
\xi_{L}= \begin{cases}n-2, & \text { if } L=S(n), \\ n-3, & \text { if } H(n) .\end{cases}
$$

Put

$$
\widetilde{S}(n)_{-1}=\operatorname{span}_{\mathbb{C}}\left\{\left(1-x_{1} x_{2} \cdots x_{n}\right) \partial_{i} \mid 1 \leq i \leq n\right\}, \widetilde{S}(n)_{i}=S(n)_{i} \text { for } i>-1 .
$$

Then $\widetilde{S}(n)$ becomes a $\mathbb{Z}_{n}$-graded Lie superalgebra: $\widetilde{S}(n)=\bigoplus_{i=-1}^{\xi_{\widetilde{S}}} \widetilde{S}(n)_{i}$, where $\xi_{\widetilde{S}}=n-2$. The 0-degree components of these superalgebras are classical Lie algebras:

$$
W(n)_{0} \cong \mathfrak{g l}(n), S(n)_{0}=\widetilde{S}(n)_{0} \cong \mathfrak{s l}(n), H(n)_{0} \cong \mathfrak{s o}(n)
$$

Note that the -1-degree components of these superalgebras is the dual module of natural module of the 0-degree components.

Let $L=\oplus_{i \in \mathbb{Z}} L_{i}$ be a $\mathbb{Z}$-graded Lie superalgbra, $H_{L}$ be the standard Cartan subalgebra of $L, \theta \in H_{L}^{*}$ be the zero weight, $\Delta^{L}$ be the root system of $L$ and $\Delta_{i}^{L}$ be the root system of $L_{i}$. If $x \in L_{\varepsilon}$ (resp. $x \in L_{i}$ ), where $\varepsilon \in H_{L}^{*}$ and $i \in \mathbb{Z}$, then we say that $x$ is $H_{L^{*}}^{*}$ homogeneous (resp. $\mathbb{Z}$-homogeneous) of degree $\varepsilon$ (resp. $i$ ) and we write $\|x\|=\varepsilon$ (resp. $\operatorname{deg}(x)=i)$.

Let us describe the roots of Cartan type Lie superalgebras. If $L=W(n)$, we choose the standard basis $\left\{\varepsilon_{1}, \ldots, \varepsilon_{n}\right\}$ in $H_{L}^{*}$, and then

$$
\Delta^{L}=\left\{\varepsilon_{i_{1}}+\cdots+\varepsilon_{i_{k}}-\varepsilon_{i} \mid 1 \leq i_{1}<\cdots<i_{k} \leq n, 1 \leq i \leq n\right\} .
$$


The root systems of $S(n)$ and $\widetilde{S}(n)$ are obtained from the root system of $W(n)$ by removing the roots $\varepsilon_{1}+\cdots+\varepsilon_{n}-\varepsilon_{i}$, where $1 \leq i \leq n$. Finally if $L=H(n)$, then

$$
\Delta^{L}=\left\{ \pm \varepsilon_{i_{1}} \pm \cdots \pm \varepsilon_{i_{k}} \mid 1 \leq i_{1}<\cdots<i_{k} \leq\left[\frac{n}{2}\right]\right\}
$$

\section{General lemmas}

In this section, let us establish several lemmas, which will be used in proving the main result for this paper. Put $\mathcal{C}=\sum_{i=1}^{n} x_{i} \partial_{i}$ and $\widetilde{H}(n)=\left\{\mathrm{D}_{\mathrm{H}}(f) \mid f \in \Lambda(n)\right\}$. By [11], we have the following Lemma.

Lemma 3.1. Let $L$ be a Cartan type Lie superalgebra. Then $\operatorname{Der} L=\operatorname{ad} L^{\prime}$, where

$$
L^{\prime} \cong \begin{cases}L, & \text { if } L=W(n), \widetilde{S}(n), \\ L \oplus \mathbb{C C}, & \text { if } L=S(n), \\ \widetilde{H}(n) \oplus \mathbb{C C}, & \text { if } L=H(n)\end{cases}
$$

By Lemma 3.1 and a simple computation, we shows $\Delta^{L^{\prime}}=\Delta^{L}$ for $L=W(n), S(n), \widetilde{S}(n)$ or $H(n)$.

Lemma 3.2. Let $L=H(n),-1 \leq k, l \leq \xi_{H}$ and $x \in L_{k}$. If $k+l=\xi_{H}$ and $\left[x, L_{l}\right]=0$, then $x=0$.

Proof. We write

$$
x=\sum_{1 \leq i_{1}<\cdots<i_{k+2} \leq n} a_{i_{1} \cdots i_{k+2}} \mathrm{D}_{\mathrm{H}}\left(x_{i_{1}} \cdots x_{i_{k+2}}\right) \in L_{k},
$$

where $a_{i_{1} \cdots i_{k+2}} \in \mathbb{C}$. For $1 \leq i_{1}<\cdots<i_{k+2} \leq n$, put

$$
I=\left\{i_{1}, \ldots, i_{k+2}\right\}, J=\left\{j_{1}, \ldots, j_{l+1}\right\}=\{1, \ldots, n\} \backslash I .
$$

Our discussion is divided into two cases.

Case 1. If $i_{1}^{\prime} \notin J$, then $0 \neq \mathrm{D}_{\mathrm{H}}\left(x_{i_{1}^{\prime}} x_{j_{1}} \cdots x_{j_{l+1}}\right) \in L_{l}$. So

$$
\left[\sum_{1 \leq i_{1}<\cdots<i_{k+2} \leq n} a_{i_{1} \cdots i_{k+2}} \mathrm{D}_{\mathrm{H}}\left(x_{i_{1}} \cdots x_{i_{k+2}}\right), \mathrm{D}_{\mathrm{H}}\left(x_{i_{1}^{\prime}} x_{j_{1}} \cdots x_{j_{l+1}}\right)\right]=0 .
$$

By equation (2.2) and observing the coefficients of terms $\mathrm{D}_{\mathrm{H}}\left(x_{i_{2}} \cdots x_{i_{k+2}} x_{j_{1}} \cdots x_{j_{l+1}}\right)$ in equation (3.1), we can obtain $a_{i_{1} \cdots i_{k+2}}=0$.

Case 2. If $i_{1}^{\prime} \in J$, without loss of generality we can let $i_{1}^{\prime}=j_{1}$, then $0 \neq \mathrm{D}_{\mathrm{H}}\left(x_{i_{1}} x_{j_{1}} \cdots x_{j_{l+1}}\right) \in$ $L_{l}$. So

$$
\left[\sum_{1 \leq i_{1}<\cdots<i_{k+2} \leq n} a_{i_{1} \cdots i_{k+2}} \mathrm{D}_{\mathrm{H}}\left(x_{i_{1}} \cdots x_{i_{k+2}}\right), \mathrm{D}_{\mathrm{H}}\left(x_{i_{1}} x_{j_{1}} \cdots x_{j_{l+1}}\right)\right]=0
$$

By equation (2.2) and observing the coefficients of terms $\mathrm{D}_{\mathrm{H}}\left(x_{i_{1}} \cdots x_{i_{k+2}} x_{j_{2}} \cdots x_{j_{l+1}}\right)$ in equation (3.2) we can obtain $a_{i_{1} \cdots i_{k+2}}=0$.

In summary, we have $x=0$. 
For a nonempty subset $V$ of a Lie superalgebra $L$, we write $\operatorname{alg}(V)$ for subalgebra of $L$, which generated by $V$. By a direct calculation, we have the following lemma.

Lemma 3.3. Let $L$ be a Cartan type Lie superalgebra. Then the following properties hold:

(1) $\left[L_{-1}, L_{i}\right]=L_{i-1}$.

(2) $\operatorname{alg}\left(L_{-1} \oplus L_{0} \oplus L_{1}\right)=L$.

(3) $L^{\prime}$ is transitive, that is $a \in \oplus_{i \geq 0} L_{i}^{\prime}$ and $\left[a, L_{-1}^{\prime}\right]=0$, then $a=0$.

(4) $L$ is irreducible, that is $L_{-1}$ is irreducible $L_{0}$-module.

Suppose $L$ is a Cartan type Lie superalgebra. Since $L$ has the weight space decompositions and $\mathbb{Z}$-grading, of course $\operatorname{BDer}(L)$ inherits a $H_{L}^{*}$-grading and $\mathbb{Z}$-grading from $L$ as above, that is

$$
\operatorname{BDer}(L)=\bigoplus_{\varepsilon \in H_{L}^{*}} \operatorname{BDer}(L)_{\varepsilon}, \operatorname{BDer}(L)=\bigoplus_{i \in \mathbb{Z}} \operatorname{BDer}(L)_{i}
$$

where

$$
\begin{gathered}
\operatorname{BDer}(L)_{\varepsilon}=\left\{f \in \operatorname{BDer}(L) \mid f\left(L_{\eta}, L_{\zeta}\right) \subseteq L_{\varepsilon+\eta+\zeta}, \forall \eta, \zeta \in H_{L}^{*}\right\}, \\
\operatorname{BDer}(L)_{i}=\left\{f \in \operatorname{BDer}(L) \mid f\left(L_{j}, L_{k}\right) \subseteq L_{i+j+k}, \forall j, k \in \mathbb{Z}\right\} .
\end{gathered}
$$

As the proof of the Lemma 3.3 in [18, p97], we can prove the following Lemma, which is useful to investigate the super-biderivations of Cartan type Lie superalgebra.

Lemma 3.4. Let $L$ be a Cartan type Lie superalgebra and $f \in \operatorname{BDer}(L)$. Then there are linear maps $\phi_{f}$ and $\psi_{f}$ from $L$ into $L^{\prime}$ such that

$$
f(x, y)=\left[\phi_{f}(x), y\right]=\left[x, \psi_{f}(y)\right]
$$

for all $x, y \in L$. Furthermore, we have the following properties hold:

(1) If $f \in \operatorname{BDer}(L)_{\varepsilon}$, then $\left\|\phi_{f}\right\|=\left\|\psi_{f}\right\|=\varepsilon$.

(2) If $f \in \operatorname{BDer}(L)_{i}$, then $\operatorname{deg}\left(\phi_{f}\right)=\operatorname{deg}\left(\psi_{f}\right)=i$.

By Lemmas 3.3 and 3.4 we have the following two lemmas.

Lemma 3.5. Let $L$ be a Cartan type Lie superalgebra and $f \in \operatorname{BDer}(L)$. If $\phi_{f}\left(L_{-1} \oplus L_{\xi_{L}}\right)=$ 0 or $\psi_{f}\left(L_{-1} \oplus L_{\xi_{L}}\right)=0$, then $f$ is zero.

Proof. We only consider the case $\phi_{f}\left(L_{-1} \oplus L_{\xi_{L}}\right)=0$. The other case may be treated in a similar way. By Lemma 3.4, we have $f(x, y)=\left[\phi_{f}(x), y\right]=0$ for all $x \in L_{-1}$ and $y \in L$. Then by Lemma 3.3 (1) and (2.1) we can prove the following fact: If $f(x, y)=0$ for all $x \in L_{i}$ and $y \in L$, then $f(z, y)=0$ for all $z \in L_{i-1}$ and $y \in L$. By Lemma 3.4, we have $f(x, y)=\left[\phi_{f}(x), y\right]=0$ for all $x \in L_{\xi_{L}}$ and $y \in L$. This together with the above fact implies that $f$ is zero.

Lemma 3.6. Let $L$ be a Cartan type Lie superalgebra and $f \in \operatorname{BDer}(L)$. If $\phi_{f}\left(L_{-1} \oplus L_{0} \oplus\right.$ $\left.L_{1}\right)=0$ or $\psi_{f}\left(L_{-1} \oplus L_{0} \oplus L_{1}\right)=0$, then $f$ is zero.

Proof. We only consider the case $\phi_{f}\left(L_{-1} \oplus L_{0} \oplus L_{1}\right)=0$. The other case may be treated in a similar way. Then by Lemma 3.4, we have $f(x, y)=0$ for all $x \in L_{-1} \oplus L_{0} \oplus L_{1}$ and $y \in L$. By Lemma $3.3(2)$ and (2.1), we have $f(z, y)=0$ for all $z, y \in L$. Then $f$ is zero.

The following Lemma is given by Theorem 2.4 in [12].

Lemma 3.7. Let $L$ be a finite-dimensional simple Lie algebra over $\mathbb{C}$. Then every biderivation of $L$ is inner. 


\section{Super-biderivations of $W(n), S(n)$ and $\widetilde{S}(n)$}

In this section we shall characterize the super-biderivations Lie superalgebras of $W(n), S(n)$ and $\widetilde{S}(n)$. Let $L=W(n), S(n)$ or $\widetilde{S}(n)$. For $\varepsilon=\sum_{i=1}^{n} k_{i} \varepsilon_{i} \in H_{L}^{*}$, we write $l(\varepsilon)=\sum_{i=1}^{n} k_{i}$. Denote

$$
A+B=\{\alpha+\beta \mid \alpha \in A, \beta \in B\},
$$

where $A, B \subseteq H_{L}^{*}$. Then we have the following super-biderivations vanishing Proposition.

Proposition 4.1. Let $L=W(n), S(n)$ or $\widetilde{S}(n)$ and $f \in \operatorname{BDer}(L)_{\varepsilon}$, where $\varepsilon \in H_{L}^{*}$. If $\varepsilon \neq 0$, then $f$ is zero.

Proof. Let $\varepsilon=\sum_{i=1}^{n} k_{i} \varepsilon_{i}$, where $k_{i} \in \mathbb{C}$. Our discussion is divided into five cases.

Case 1. Suppose $k_{i} \notin \mathbb{Z}$ for some $1 \leq i \leq n$. In this case, we have $\left(\varepsilon+\Delta^{L}\right) \cap \Delta^{L^{\prime}}=\emptyset$. Since $\left\|\phi_{f}(x)\right\| \in \varepsilon+\Delta^{L}$ for all $x \in L$, then $\phi_{f}(x)=0$. By Lemma 3.4, we can obtain $f$ is zero.

Case 2. Suppose $k_{i} \in \mathbb{Z}$ for all $1 \leq i \leq n$ and $k_{j}<0$ for some $1 \leq j \leq n$. In this case, we have $\varepsilon-\varepsilon_{j} \notin \Delta^{L}$ for all $1 \leq j \leq n$. Note that $\phi_{f}\left(\partial_{j}\right), \psi_{f}\left(\partial_{j}\right) \in L_{\varepsilon-\varepsilon_{j}}^{\prime}$. Then $\phi_{f}\left(\partial_{j}\right)=\psi_{f}\left(\partial_{j}\right)=0$ for all $1 \leq j \leq n$. Next, Our discussion is divided into two subcases.

Subcase 2.1. Suppose $\varepsilon \notin-\Delta_{\xi_{L}}^{L}+\Delta_{-1}^{L}$. In this case, we have $\left(\varepsilon+\Delta_{\xi_{L}}^{L}\right) \cap \Delta_{-1}^{L^{\prime}}=\emptyset$. Since

$$
\left\|\phi_{f}\left(L_{\xi_{L}}\right)\right\|,\left\|\psi_{f}\left(L_{\xi_{L}}\right)\right\| \in \varepsilon+\Delta_{\xi_{L}}^{L}
$$

then $\left\|\phi_{f}\left(L_{\xi_{L}}\right)\right\|,\left\|\psi_{f}\left(L_{\xi_{L}}\right)\right\| \notin \Delta_{-1}^{L_{-1}^{\prime}}$. Therefore $\phi_{f}\left(L_{\xi_{L}}\right), \psi_{f}\left(L_{\xi_{L}}\right) \in \oplus_{i \geq 0} L_{i}^{\prime}$. By Lemma 3.4. we have

$$
0=\left[\phi_{f}\left(\partial_{j}\right), L_{\xi_{L}}\right]=\left[\partial_{j}, \psi_{f}\left(L_{\xi_{L}}\right)\right]
$$

for all $1 \leq j \leq n$. By Lemma 3.3 (3), we have $\psi_{f}\left(L_{\xi_{L}}\right)=0$. By Lemma 3.5 we have $f$ is zero.

Subcase 2.2. Suppose $\varepsilon \in-\Delta_{\xi_{L}}^{L}+\Delta_{-1}^{L}$. By direct calculation, we have

$$
-\Delta_{\xi_{L}}^{L}+\Delta_{-1}^{L}= \begin{cases}\left\{-\sum_{i=1}^{n} \varepsilon_{i}+\varepsilon_{j}-\varepsilon_{l} \mid 1 \leq j, l \leq n\right\}, & \text { if } L=W(n), \\ \left\{-\sum_{j \neq i=1}^{n} \varepsilon_{i}+\varepsilon_{k}-\varepsilon_{l} \mid 1 \leq j, k, l \leq n\right\}, & \text { if } L=S(n), \widetilde{S}(n) .\end{cases}
$$

Then for $\varepsilon \in-\Delta_{\xi_{L}}^{L}+\Delta_{-1}^{L}$, we have

$$
l(\varepsilon)= \begin{cases}-n, & \text { if } L=W(n), \\ -n+1, & \text { if } L=S(n), \widetilde{S}(n) .\end{cases}
$$

Sequentially, we have

$$
l\left(\left\|\phi_{f}(x)\right\|\right)=l(\varepsilon)+l(\|x\|)= \begin{cases}-n, & \text { if } L=W(n), \\ -n+1, & \text { if } L=S(n), \widetilde{S}(n)\end{cases}
$$

for all $x \in L_{0}$. Note that $l(\alpha) \geq-1$ for all $\alpha \in \Delta^{L^{\prime}}$ and $n \geq 4$. Therefore, $\phi_{f}(x)=0$, that is $\phi_{f}\left(L_{0}\right)=0$. Similarly, we can prove that $\phi_{f}\left(L_{1}\right)=0$. By Lemma 3.6. we can obtain $f$ is zero.

Case 3. Suppose $k_{i} \in \mathbb{Z}$ for all $1 \leq i \leq n$ and $k_{j} \geq 3$ for some $1 \leq j \leq n$. Note that for any $\alpha=\sum_{i=1}^{n} l_{i} \varepsilon_{i} \in \Delta^{L}$, we have $l_{j} \in\{-1,0,1\}$. Then $k_{j}+l_{j} \geq 2$. So $\varepsilon+\alpha \notin \Delta^{L^{\prime}}$, that is $\left(\varepsilon+\Delta^{L}\right) \cap \Delta^{L^{\prime}}=\emptyset$. Since $\left\|\phi_{f}(x)\right\| \in \varepsilon+\Delta^{L}$ for all $x \in L$, then $\phi_{f}(x)=0$. By Lemma 3.4. we can obtain $f$ is zero. 
Case 4. Suppose $k_{i} \in \mathbb{Z}$ for all $1 \leq i \leq n$ and $k_{p}=k_{q}=2$ for some $1 \leq p \neq q \leq n$. Note that for any $\alpha=\sum_{i=1}^{n} l_{i} \varepsilon_{i} \in \Delta^{L}$, we have $l_{p}, l_{q} \in\{-1,0,1\}$, but $l_{p}$ and $l_{q}$ are not all equal to -1 at the same time. Then $k_{p}+l_{p} \geq 2$ or $k_{q}+l_{q} \geq 2$. So $\varepsilon+\alpha \notin \Delta^{L^{\prime}}$, that is $\left(\varepsilon+\Delta^{L}\right) \cap \Delta^{L^{\prime}}=\emptyset$. Since $\left\|\phi_{f}(x)\right\| \in \varepsilon+\Delta^{L}$ for all $x \in L$, then $\phi_{f}(x)=0$. By Lemma 3.4. we can obtain $f$ is zero.

Case 5. Suppose $\varepsilon=\sum_{i=1}^{n} k_{i} \varepsilon_{i}$, where $k_{i} \in\{0,1,2\}$ and at most one of $k_{i}$ is equal to 2. Without loss of generality, we can write $\varepsilon=a \varepsilon_{1}+\varepsilon_{2}+\cdots+\varepsilon_{i}$, where $1 \leq i \leq n$ and $a \in\{1,2\}$. Since $\varepsilon \neq \theta, l(\varepsilon)>0$. Then

$$
l(\varepsilon+\alpha)> \begin{cases}n-1, & \text { if } L=W(n) \\ n-2, & \text { if } L=S(n), \widetilde{S}(n)\end{cases}
$$

for all $\alpha \in \Delta_{\xi_{L}}^{L}$. Note that

$$
l(\beta) \leq \begin{cases}n-1, & \text { if } L=W(n) \\ n-2, & \text { if } L=S(n), \widetilde{S}(n)\end{cases}
$$

for all $\beta \in \Delta^{L^{\prime}}$. Therefore, $\left(\varepsilon+\Delta_{\xi_{L}}^{L}\right) \cap \Delta^{L^{\prime}}=\emptyset$. Then $\phi_{f}\left(L_{\xi_{L}}\right)=\psi_{f}\left(L_{\xi_{L}}\right)=0$. Next, Our discussion is divided into two subcases.

Subcase 5.1. Suppose $\varepsilon=2 \varepsilon_{1}+\varepsilon_{2}+\cdots+\varepsilon_{i}$. In this case, we have $\phi_{f}\left(H_{L}\right)=\phi_{f}\left(H_{L}\right)=0$. By Lemma 3.4, we have

$$
\begin{aligned}
0 & =\left[\phi_{f}(h), \partial_{l}-\delta_{L, \widetilde{S}} x_{1} x_{2} \cdots x_{n} \partial_{l}\right]=\left[h, \psi_{f}\left(\partial_{l}-\delta_{L, \widetilde{S}} x_{1} x_{2} \cdots x_{n} \partial_{l}\right)\right] \\
& =\left(2 \varepsilon_{1}+\varepsilon_{2}+\cdots+\varepsilon_{i}-\varepsilon_{l}\right)(h) \psi_{f}\left(\partial_{l}-\delta_{L, \widetilde{S}} x_{1} x_{2} \cdots x_{n} \partial_{l}\right)
\end{aligned}
$$

for all $h \in H_{L}$. Since $2 \varepsilon_{1}+\varepsilon_{2}+\cdots+\varepsilon_{i}-\varepsilon_{l} \neq \theta$, there exists $h \in H_{L}$ such that $\left(2 \varepsilon_{1}+\varepsilon_{2}+\cdots+\right.$ $\left.\varepsilon_{i}-\varepsilon_{l}\right)(h) \neq 0$. By (4.1), we have $\psi_{f}\left(\partial_{l}-\delta_{L, \widetilde{S}_{1}} x_{1} x_{2} \cdots x_{n} \partial_{l}\right)=0$, that is $\psi_{f}\left(L_{-1}\right)=0$. By Lemma 3.5, we have $f$ is zero.

Subcase 5.2. Suppose $\varepsilon=\varepsilon_{1}+\varepsilon_{2}+\cdots+\varepsilon_{i}$. In this case, we have

$$
\phi_{f}\left(x_{1} \partial_{j}\right), \psi_{f}\left(x_{1} \partial_{j}\right) \in L_{2 \varepsilon_{1}+\varepsilon_{2}+\cdots+\varepsilon_{i}-\varepsilon_{j}}^{\prime}=0
$$

for all $j \neq 1$. Since $\phi_{f}\left(\partial_{1}-\delta_{L, \widetilde{S}} x_{1} x_{2} \cdots x_{n} \partial_{1}\right) \in L_{\varepsilon_{2}+\cdots+\varepsilon_{i}}^{\prime}$ and

$$
L_{\varepsilon_{2}+\cdots+\varepsilon_{i}}^{\prime}=\operatorname{span}_{\mathbb{C}}\left\{x_{2} \cdots x_{i} x_{j} \partial_{j} \mid j=1, i+1, \ldots, n\right\} \cap L^{\prime},
$$

we can write

$$
\phi_{f}\left(\partial_{1}-\delta_{L, \widetilde{S}} x_{1} x_{2} \cdots x_{n} \partial_{1}\right)=a_{1} x_{2} \cdots x_{i} x_{1} \partial_{1}+\sum_{j=i+1}^{n} a_{j} x_{2} \cdots x_{i} x_{j} \partial_{j},
$$

where $a_{1}, a_{j} \in \mathbb{C}$. By Lemma 3.4, we have

$$
\begin{aligned}
0 & =\left[\partial_{1} \delta_{L, \widetilde{S}} x_{1} x_{2} \cdots x_{n} \partial_{1}, \psi_{f}\left(x_{1} \partial_{2}\right)\right]=\left[\phi_{f}\left(\partial_{1}-\delta_{L, \widetilde{S}} x_{1} x_{2} \cdots x_{n} \partial_{1}\right), x_{1} \partial_{2}\right] \\
& =\left[a_{1} x_{2} \cdots x_{i} x_{1} \partial_{1}+\sum_{j=i+1}^{n} a_{j} x_{2} \cdots x_{i} x_{j} \partial_{j}, x_{1} \partial_{2}\right] \\
& =a_{1} x_{2} \cdots x_{i} x_{1} \partial_{2}-\sum_{j=i+1}^{n} a_{j} x_{1} x_{3} \cdots x_{i} x_{j} \partial_{j}
\end{aligned}
$$


Then $a_{1}=a_{i+1}=\cdots=a_{n}=0$, that is $\phi_{f}\left(\partial_{1}-\delta_{L, \widetilde{S}} x_{1} x_{2} \cdots x_{n} \partial_{1}\right)=0$. Similarly, we can prove that $\psi_{f}\left(\partial_{1}-\delta_{L, \widetilde{S}} x_{1} x_{2} \cdots x_{n} \partial_{1}\right)=0$. Let $h \in H_{L}$. Since $\phi_{f}(h) \in L_{\varepsilon_{1}+\cdots+\varepsilon_{i}}^{\prime}$ and

$$
L_{\varepsilon_{1}+\cdots+\varepsilon_{i}}^{\prime}=\operatorname{span}_{\mathbb{C}}\left\{x_{1} \cdots x_{i} x_{j} \partial_{j} \mid j=i+1, \ldots, n\right\} \cap L^{\prime},
$$

we can write

$$
\phi_{f}(h)=\sum_{j=i+1}^{n} a_{j} x_{2} \cdots x_{i} x_{j} \partial_{j},
$$

where $a_{j} \in \mathbb{C}$. By Lemma 3.4, we have

$$
\begin{aligned}
0 & =\left[\phi_{f}\left(\partial_{1}-\delta_{L, \widetilde{S}} x_{1} x_{2} \cdots x_{n} \partial_{1}\right), h\right]=\left[\partial_{1}-\delta_{L, \widetilde{S}} x_{1} x_{2} \cdots x_{n} \partial_{1}, \psi_{f}(h)\right] \\
& =\left[\partial_{1}-\delta_{L, \widetilde{S}} x_{1} x_{2} \cdots x_{n} \partial_{l}, \sum_{j=i+1}^{n} a_{j} x_{1} \cdots x_{i} x_{j} \partial_{j}\right] \\
& =\sum_{j=i+1}^{n} a_{j} x_{2} x_{3} \cdots x_{i} x_{j} \partial_{j} .
\end{aligned}
$$

Then $a_{i+1}=\cdots=a_{n}=0$, that is $\psi_{f}(h)=0$. Similarly, we can prove that $\phi_{f}(h)=0$. Note that $\phi_{f}\left(\partial_{l}\right), \psi_{f}\left(\partial_{l}\right) \in L_{\varepsilon_{1}+\cdots+\varepsilon_{i}-\varepsilon_{l}}^{\prime}$ and there exists $h \in H_{L}$ such that $\left(\varepsilon_{1}+\cdots+\varepsilon_{i}-\varepsilon_{l}\right)(h) \neq$ 0 for all $l \neq 1$. By Lemma 3.4, we have

$$
\begin{aligned}
0 & =\left[\partial_{l}-\delta_{L, \widetilde{S}} x_{1} x_{2} \cdots x_{n} \partial_{l}, \psi_{f}(h)\right]=\left[\phi_{f}\left(\partial_{l}-\delta_{L, \widetilde{S}} x_{1} x_{2} \cdots x_{n} \partial_{l}\right), h\right] \\
& =-\left(\varepsilon_{1}+\cdots+\varepsilon_{i}-\varepsilon_{l}\right)(h) \phi_{f}\left(\partial_{l}-\delta_{L, \widetilde{S}} x_{1} x_{2} \cdots x_{n} \partial_{l}\right) .
\end{aligned}
$$

Then $\phi_{f}\left(\partial_{l}-\delta_{L, \widetilde{S}} x_{1} x_{2} \cdots x_{n} \partial_{l}\right)=0$ for all $l \neq 1$. In summary, $\phi\left(L_{-1}\right)=0$. By Lemma 3.5. we have $f=0$.

Proposition 4.2. Let $L=W(n), S(n)$ or $\widetilde{S}(n)$. If $f \in \operatorname{BDer}(L)_{\theta}$, then $f$ is inner, that is there is $\lambda \in \mathbb{C}$ such that $f(x, y)=\lambda[x, y]$ for all $x, y \in L$.

Proof. Let $1 \leq i \leq n$ and $\varepsilon \in \Delta_{\xi_{L}}^{L^{\prime}}$. Since $\operatorname{dim} L_{-\varepsilon_{i}}^{\prime}=1$ and $\operatorname{dim}\left(L_{\xi_{L}}^{\prime}\right)_{\varepsilon}=1$, then we have

$$
\begin{aligned}
& \phi_{f}\left(\partial_{i}-\delta_{L, \widetilde{S}} x_{1} x_{2} \cdots x_{n} \partial_{i}\right)=\lambda_{i}\left(\partial_{i}-\delta_{L, \widetilde{S}} x_{1} x_{2} \cdots x_{n} \partial_{i}\right), \\
& \psi_{f}\left(\partial_{i}-\delta_{L, \widetilde{S}} x_{1} x_{2} \cdots x_{n} \partial_{i}\right)=\beta_{i}\left(\partial_{i}-\delta_{L, \widetilde{S}} x_{1} x_{2} \cdots x_{n} \partial_{i}\right),
\end{aligned}
$$

$\phi_{f}(x)=\lambda_{x} x$ and $\psi_{f}(x)=\beta_{x} x$, where $\lambda_{i}, \beta_{i}, \lambda_{x}, \beta_{x} \in \mathbb{C}$ and $x \in\left(L_{\xi_{L}}^{\prime}\right)_{\varepsilon}$. By Lemma 3.4. we have

$$
\left[\phi_{f}\left(\partial_{i}-\delta_{L, \widetilde{S}} x_{1} x_{2} \cdots x_{n} \partial_{i}\right), x\right]=\left[\partial_{i}-\delta_{L, \widetilde{S}} x_{1} x_{2} \cdots x_{n} \partial_{i}, \psi_{f}(x)\right] .
$$

Then $\lambda_{i}=\beta_{x}$. Similarly, we have $\beta_{i}=\lambda_{x}$. Therefore, $\lambda_{1}=\cdots=\lambda_{n}$ and $\beta_{1}=\cdots=\beta_{n}$, denoted $\lambda$ and $\beta$, respectively. We put $\lambda=\lambda_{1}$ and $\beta=\beta_{1}$. Let $y \in \sum_{j \geq 0} L_{j}$ and $1 \leq i \leq n$, 
then

$$
\begin{aligned}
f\left(\partial_{i}-\delta_{L, \widetilde{S}} x_{1} x_{2} \cdots x_{n} \partial_{i}, y\right) & =\left[\phi_{f}\left(\partial_{i}-\delta_{L, \widetilde{S}_{1}} x_{1} x_{2} \cdots x_{n} \partial_{i}\right), y\right] \\
& =\lambda\left[\partial_{i}-\delta_{L, \widetilde{S}} x_{1} x_{2} \cdots x_{n} \partial_{i}, y\right] \\
& =\left[\partial_{i}-\delta_{L, \widetilde{S}} x_{1} x_{2} \cdots x_{n} \partial_{i}, \psi_{f}(y)\right]
\end{aligned}
$$

Then

$$
\left[\partial_{i}-\delta_{L, \widetilde{S}} x_{1} x_{2} \cdots x_{n} \partial_{i}, \lambda y-\psi_{f}(y)\right]=0
$$

for all $1 \leq i \leq n$. Note that $\psi_{f}\left(\oplus_{j \geq 0} L_{j}\right) \subseteq \oplus_{j \geq 0} L_{j}^{\prime}$, So by Lemma 3.3 (3), we have $\psi_{f}(y)=\lambda y$. Similarly, we have $\phi_{f}(y)=\beta y$. By Lemma 3.4 we have

$$
f\left(x_{1} \partial_{2}, x_{2} \partial_{1}\right)=\left[\phi_{f}\left(x_{1} \partial_{2}\right), x_{2} \partial_{1}\right]=\beta\left[x_{1} \partial_{2}, x_{2} \partial_{1}\right]=\left[x_{1} \partial_{2}, \psi_{f}\left(x_{2} \partial_{1}\right)\right]=\lambda\left[x_{1} \partial_{2}, x_{2} \partial_{1}\right] .
$$

Then $\lambda=\beta$. In summary, $\phi_{f}(x)=\lambda x$ for all $x \in L$. That is $f$ is inner.

By Propositions 4.1 and 4.2 we have the following theorem.

Theorem 4.3. Let $L=W(n), S(n)$ or $\widetilde{S}(n)$. Then

$$
\operatorname{BDer}(L)=\operatorname{IBDer}(L) \text {. }
$$

\section{Super-biderivations of $H(n)$}

In this section we shall characterize the super-biderivations of $H(n)$. We have the following super-biderivations vanishing Proposition.

Proposition 5.1. Let $L=H(n)$ and $f \in \operatorname{BDer}(L)_{i}$, where $i \in \mathbb{Z}$. If $i \neq 0$, then $f$ is zero.

Proof. Our discussion is divided into three cases.

Case 1. Suppose $i<-\xi_{H}$. Since $\xi_{H}=n-2$ and $n>4, \phi_{f}\left(L_{-1} \oplus L_{0} \oplus L_{1}\right)=0$. By Lemma 3.6, we have $f=0$.

Case 2. Suppose $-\xi_{H} \leq i \leq-1$. In this case, we have $\phi_{f}\left(L_{-1}\right)=\psi_{f}\left(L_{-1}\right)=0$ and $\psi_{f}\left(L_{\xi_{H}}\right) \subseteq \oplus_{j \geq 0} L_{j}^{\prime}$. Let $x \in L_{\xi_{H}}$ and $1 \leq j \leq n$. Then by Lemma 3.4, we have

$$
0=\left[\phi_{f}\left(\partial_{j}\right), x\right]=f\left(\partial_{j}, x\right)=\left[\partial_{j}, \psi_{f}(x)\right] .
$$

By Lemma 3.3 (3), we have $\psi_{f}(x)=0$, that is $\psi_{f}\left(L_{\xi_{H}}\right)=0$. By Lemma 3.5, we have $f=0$.

Case 3. Suppose $i \geq 1$. In this case, we have

$$
\phi_{f}\left(L_{\xi_{H}-i+1} \oplus \cdots \oplus L_{\xi_{H}}\right)=\psi_{f}\left(L_{\xi_{H}-i+1} \oplus \cdots \oplus L_{\xi_{H}}\right)=0 .
$$

Let $x \in L_{\xi_{H}-i+1}$ and $1 \leq j \leq n$. By Lemma 3.4 we have

$$
0=\left[\partial_{j}, \psi_{f}(x)\right]=f\left(\partial_{j}, x\right)=\left[\phi_{f}\left(\partial_{j}\right), x\right],
$$

that is $\left[\phi_{f}\left(\partial_{j}\right), L_{\xi_{H}-i+1}\right]=0$. Note that $\phi_{f}\left(\partial_{j}\right) \in L_{i-1}^{\prime}$. By Lemma 3.2, we have $\phi_{f}\left(\partial_{j}\right)=0$. That is $\phi_{f}\left(L_{-1}\right)=0$. By Lemma 3.5, we have $f$ is zero.

Proposition 5.2. Let $L=H(n)$. If $f \in \operatorname{BDer}(L)_{0}$, then $f$ is inner, that is there is $\lambda \in \mathbb{C}$ such that $f(x, y)=\lambda[x, y]$ for all $x, y \in L$. 
Proof. In this case, we have $\left.f\right|_{L_{0} \times L_{0}}$ is super-biderivation of $L_{0}$. By Lemma 3.7 we can write $\phi_{f}(x)=\psi_{f}(x)=\lambda x$ for all $x \in L_{0}$. By Lemma 3.4, we have

$$
\lambda[x, y]=\left[\phi_{f}(y), y\right]=f(x, y)=\left[x, \psi_{f}(y)\right]
$$

for all $x \in L_{0}$ and $y \in L_{-1}$. Then $\left[L_{0}, \lambda y-\psi_{f}(y)\right]=0$. By Lemma 3.3 (4), we have $\psi_{f}(y)=\lambda y$ for all $y \in L_{-1}$. By Lemma 3.4, we have

$$
\lambda[x, y]=\left[\phi_{f}(x), y\right]=f(x, y)=\left[x, \psi_{f}(y)\right]
$$

for all $x \in L_{-1}$ and $y \in \oplus_{i \geq 1} L_{i}$. Then $\left[L_{-1}, \lambda y-\psi_{f}(y)\right]=0$. By Lemma $3.3(3)$, we have $\psi_{f}(y)=\lambda y$. In summary, $\psi_{f}(x)=\lambda x$ for all $x \in L$. That is $f$ is inner.

By Propositions 5.1 and 5.2 we have the following theorem.

Theorem 5.3. Let $L=H(n)$. Then

$$
\operatorname{BDer}(L)=\operatorname{IBDer}(L)
$$

\section{References}

[1] Brešar, M.: On generalized biderivations and related maps. J. Algebra 172(3), 764786 (1995)

[2] Chen, Z.: Biderivations and linear commuting maps on simple generalized Witt algebras over a field, Elec J. Linear algebra 31, 1-12 (2016)

[3] Cheng, X., Wang, M., Sun, J., Zhang, H.: Biderivations and linear commuting maps on the Lie algebra gca, Linear Multilinear Algebra 65(12), 1-11(2017)

[4] Du, Y., Wang, Y.: Biderivations of generalized matrix algebras. Linear Algebra Appl. 438(11), 4483-4499 (2013)

[5] Fan, G., Dai, X.: Super-biderivations of Lie superalgebras, Linear Multilinear Algebra 65(1), 58-66 (2017)

[6] Ghosseiri, N.: On biderivations of upper triangular matrix rings. Linear Algebra Appl. 438(1), 250-260 (2013)

[7] Han, X., Wang, D., Xia, C.: Linear commuting maps and biderivations on the Lie algebras $W(a, b)$. J. Lie theory 26(3), 777-786 (2016)

[8] Humphreys, J.: Introduction to Lie algebras and representation theory, Springer Science and Business Media, (1972)

[9] Kac, V.: Lie superalgebras, Adv. Math. 26, 8-96 (1977)

[10] Li, W., Tang, X., Yuan, J.: Super-biderivations of classical simple Lie superalgebras, Aequat. Math. 92, 91-109 (2018)

[11] Scheunert, M.: Theory of Lie superalgebras, in: Lecture Notes in Math. 716, SpringerVerlag, (1979)

[12] Tang, X.: Biderivations of finite dimensional complex simple Lie algebras, Linear Multilinear Algebra 66(3), 1-10 (2016) 
[13] Tang, X.: Biderivations, linear commuting maps and commutative post-Lie algebra structures on W-algebras, Comm. Algebra 45(12), 5252-5261 (2017)

[14] Wang, D., Yu, X., Chen, Z.: Biderivations of parabolic subalgebras of simple Lie algebras, Comm. Algebra 39(11), 4097-4104 (2011)

[15] Wang, D., Yu, X.: Biderivations and linear commuting maps on the SchrödingerVirasoro Lie algebra, Comm. Algebra 41(6), 2166-2173 (2013)

[16] Xu, H., Wang, L.: The properties of biderivations on Heisenberg superalgebras, Mathematica Aeterna 5(2), 285-291(2015)

[17] Xia, C., Wang, D., Han, X.: Linear Super-Commuting Maps and Super-Biderivations on the Super-Virasoro Algebras, Comm. Algebra 44(22), 5342-5350 (2016)

[18] Yuan, J., Tang, X.: Super-biderivations of classical simple Lie superalgebras, Aequat. Math. 92, 91-109 (2018) 